Check for updates

Cite this: RSC Adv., 2019, 9, 19347

Received 30th March 2019

Accepted 6th June 2019

DOI: $10.1039 / c 9 r a 02401 \mathrm{~h}$

rsc.li/rsc-advances

\section{A smart fluorescent biosensor for the highly sensitive detection of BRCA1 based on a 3D DNA walker and ESDR cascade amplification $\dagger$}

\author{
Hongmin Ma, \$ Bin Guo, \$ Xiaoyu Yan, Tong Wang, Haiying Que, Xiufeng Gan, Ping Liu \\ and Yurong Yan (iD)*
}

Nucleic acid analysis plays an important role in the diagnosis of diseases. There is a continuous demand to develop rapid and sensitive methods for the specific detection of nucleic acids. Herein, we constructed a highly sensitive and rapid fluorescent biosensor for the detection of BRCA1 by coupling a 3D DNA walker machine with spontaneous entropy-driven strand displacement reactions (ESDRs). In this study, the 3D DNA walker machine was well activated by the target DNA; this resulted in the cyclic utilization of the target DNA and the release of intermediate DNAs. Subsequently, the free intermediate DNAs triggered the circulation process of ESDRs with the help of the assistant probe A, leading to a significant enhancement of the fluorescence intensity. Due to the robust execution of the 3D DNA walker machine and highly efficient amplification capability of ESDRs, the developed biosensing method shows a wide linear range from $0.1 \mathrm{pM}$ to $10 \mathrm{nM}$ with the detection limit as low as $41.44 \mathrm{fM}(\mathrm{S} / \mathrm{N}=3)$. Moreover, the constructed biosensor displays superior specificity and has been applied to monitor BRCA1 in complex matrices. Thus, this elaborated cascade amplification biosensing strategy provides a potential platform for the bioassays of nucleic acids and the clinical diagnosis of diseases.

\section{Introduction}

The accurate analysis of nucleic acids is fundamentally important in the diagnosis, ${ }^{1}$ therapeutics ${ }^{2}$ and biological differentiation $^{3}$ of diseases. However, low concentrations of target DNA or RNA in biological samples present a great challenge for analytical technology. ${ }^{4,5}$ Although non-isothermal amplification techniques, such as the polymerase chain reaction (PCR), the digital polymerase chain reaction (dPCR) and so on, are among the most widely used methods for biomolecular detection, researchers continue to seek an isothermal amplification-based strategy capable of adapting to the requirements of nucleic acid detection in various environments. Therefore, various studies have been employed to explore novel biosensing methods for the precise analysis of nucleic acids. ${ }^{6,7}$ For example, Li's team established a new DNA colorimetric sensing strategy by conjugating graphene oxide with DNA-modified gold nanoparticles for the detection of DNA. ${ }^{8}$ However, these biosensing strategies

Key Laboratory of Clinical Laboratory Diagnostics (Ministry of Education), College of Laboratory Medicine, Chongqing Medical University, Chongqing 400016, China. E-mail: yanyurong163@163.com; yanyurong@cqmu.edu.cn; Fax: +86-23-68485786; Tel: +86-23-68485688

$\dagger$ Electronic supplementary information (ESI) available. See DOI: 10.1039/c9ra02401h

$\$$ These two authors contributed equally to this work. still cannot meet the needs of low-abundance target molecule detection due to their insufficient sensitivity.

To overcome this drawback, substantial signal amplification strategies, including rolling circle amplification, ${ }^{9}$ the ligase chain reaction, ${ }^{10}$ the hairpin-based amplification assay ${ }^{11,12}$ and a molecular machine-based assay, ${ }^{13,14}$ have been developed to improve the analytical performance. In particular, the development of DNA walker machines has drawn extensive attention due to their precise controllability along a designated trace..$^{15,16}$ However, conventional one-dimensional (1D) or twodimensional (2D) DNA walker machines usually have common defects such as low mobility and less cargo payload. ${ }^{17,18}$ Impressively, three-dimensional (3D) DNA machines, with effective cargo-loading capacity and rapid target-recycling kinetics, show more prominent amplification performance. Furthermore, via highly efficient catalysis of proteases, the multistep movement of the cargo instead of a single step can be achieved in the 3D DNA machine. ${ }^{19,20}$ For example, Li's group designed a nicking endonuclease-driven 3D DNA walker machine and explored its isothermal, rapid and homogeneous advantages to detect specific nucleic acids. ${ }^{21}$ However, only a single recycling amplification strategy was adopted in the design, which limited the signal enhancement.

Due to the abovementioned issues, a number of cascade signal amplification strategies have been extensively developed to further improve the performance of detection. ${ }^{22,23}$ In 2017, Chen's group developed an electrochemiluminescence (ECL) 
biosensor based on a DNA walker and rolling circle amplification (RCA), achieving ultrasensitive and specific detection of the K-ras gene. ${ }^{24}$ Subsequently, Jiang et al. constructed a fluorescent biosensor based on a DNAzyme-powered three-dimensional DNA walker and hybridization chain reaction (HCR), achieving enzyme-free and label-free detection of miRNAs. ${ }^{25}$ In these studies, various cascaded circuit products from the first DNA-based circuit served as the input/trigger for the second DNA-based circuit and resulted in further signal enhancement. It is undeniable that these strategies have improved the sensitivity of detection to some extent. However, these methods also have some limitations. For instance, the RCA reaction requires the participation of multiple proteases and time-consuming template preparation, which limit the application of RCA in practice. The hybridization chain reaction, involving complicated secondary structures of hairpin and laborious sequence design, usually produces a higher background signal. ${ }^{\mathbf{2 6 , 2 7}}$

Compared to the hairpin-based designs, entropy-driven strand displacement reactions (ESDRs) ${ }^{28-30}$ employ a series of single-stranded DNA to perform multiple hybridization and separation in the catalytic circuit via toehold-assisted strand migration. More importantly, the design of ESDRs, which does not involve complex secondary structures, is relatively simple and fast. For instance, Chen's group introduced ESDRs into a electrochemiluminescence platform to achieve the ultrasensitive detection of target DNA. ${ }^{31}$ Furthermore, due to the good biocompatibility of ESDRs, Chen and co-workers constructed a cascade reaction by coupling strand displacement amplification with ESDRs for the rapid and ultrasensitive detection of microRNA. ${ }^{32}$ Altogether, as a valuable tool for nucleic acid amplification, ESDR assays are receiving significant attention due to their inherent advantages including excellent thermostability, high amplification efficiency and robust resistance to complex environments.

Inspired by the high cargo payload ability of DNA walker machines and excellent amplification effectiveness of ESDRs, a smart fluorescence biosensing strategy was developed for nucleic acid detection using the BRCA1 gene as a model. By integrating a 3D DNA walker machine and ESDRs, the sensitivity of the DNA detection was significantly enhanced, and the signal-to-noise ratio was obviously improved. Furthermore, due to the superb discriminative ability of toehold-mediated strand displacement reaction (TMSDR), this biosensing strategy shows high specificity. To the best of our knowledge, this is the first attempt to combine a 3D DNA walker with ESDR assays for BRCA1 detection, which may present a promising tool for nucleic acid analysis in biochemical studies and clinical diagnosis.

\section{Experimental}

\subsection{Materials and reagents}

Streptavidin-magnetic beads $\left(1 \mu \mathrm{m}\right.$ diameter, $4 \mathrm{mg} \mathrm{mL}^{-1}$, an aqueous suspension containing $0.1 \%$ bovine serum albumin, $\mathrm{pH} 7.4,0.05 \%$ Tween-20, and $0.05 \% \mathrm{NaN}_{3}$ ), nicking endonuclease (Nb.BbvCI) and $10 \times$ CutSmart $^{\mathrm{TM}}$ buffer $(20 \mathrm{mM}$, Trisacetate, $500 \mathrm{mM}$ potassium acetate, $10 \mathrm{mM}$ magnesium acetate, and $100 \mu \mathrm{g} \mathrm{mL}^{-1} \mathrm{BSA}, \mathrm{pH}$ 7.9) were purchased from New England Biolabs (Beijing, China). Gold View I (GV I) $(10000 \times)$ used in this study was provided by Solarbio Co. Ltd. (Xiamen, China). The DL 200 DNA marker was ordered from Takara (Dalian, China). Salmon sperm DNA was obtained from Solarbio Inc. (Beijing, China). All oligonucleotides were synthesized and purified via HPLC by Sangon Biotechnology Co. Ltd. (Shanghai, China), and the corresponding sequences are illustrated in Table S1 (ESI). $\dagger$ All oligonucleotides were dissolved in tris-ethylenediaminetetraacetic acid (TE) buffer ( $\mathrm{pH}$ 8.0, $10 \mathrm{mM}$ Tris-HCl, $1 \mathrm{mM}$ EDTA) and stored at $-20{ }^{\circ} \mathrm{C}$ for further use.

Moreover, 0.1 M phosphate-buffered solution (PBS) included 136.89 $\mathrm{mM} \mathrm{NaCl}, 2.67 \mathrm{mM} \mathrm{KCl}, 8.10 \mathrm{mM} \mathrm{Na}_{2} \mathrm{HPO}_{4}$ and $1.76 \mathrm{mM}$ $\mathrm{KH}_{2} \mathrm{PO}_{4}$ (pH 7.4). The reaction buffer contained $10 \mathrm{mM}$ Tris$\mathrm{HCl}, 1 \mathrm{mM}$ EDTA and $12.5 \mathrm{mM} \mathrm{MgCl}_{2}$ (pH 8.0). Other chemicals (analytical grade) were obtained from standard reagent suppliers. Millipore-Q water ( $\geq 18 \mathrm{M} \Omega$, Milli-Q, Millipore) was used throughout the experiments.

\subsection{Instrumentations}

Fluorescence signals were obtained using the Cary Eclipse Fluorescence spectrophotometer (Agilent, California). The concentrations of DNA suspensions were measured using the NanoDrop 1000 spectrophotometer (Thermo Scientific, Wilmington, DE, USA). Gel electrophoresis was carried out using the DYY-6C electrophoresis analyzer (Liuyi Instrument Company, China), and the image was acquired using the BioRad ChemDoc XRS (Bio-Rad Laboratories, USA). Furthermore, the Bio-Rad T100 thermal cycler (Bio-Rad, USA) was used as a temperature control device in this study.

\subsection{Preparation of SDs-PWTs-SMBs}

Before use, $10 \mu \mathrm{M}$ biotin-modified DNA walker tracks (DWTs) and $10 \mu \mathrm{M}$ protecting probe (PP) were dissolved in TE buffer at equal volumes. The resulting mixture was incubated at $90{ }^{\circ} \mathrm{C}$ for $10 \mathrm{~min}$ and then gradually cooled down to room temperature to form the protected DNA walker probe (PWTs, $5 \mu \mathrm{M}$ ). Then, 12.5 $\mu \mathrm{L}$ SMBs was washed three times with PBS and then redispersed in $41.75 \mu \mathrm{L}$ of the same buffer. The PWTs and biotin-modified substrate strands of DNA (SDs) at the ratio of 1 to 20 were sufficiently mixed and then added to the SMB solution for $30 \mathrm{~min}$ at $37{ }^{\circ} \mathrm{C}$. Due to the specific interaction between streptavidin and biotin, the biotin-modified DNA were firmly bound to the streptavidin SMBs to obtain a mixing solution (PWTsSDs-SMBs) containing $75 \mathrm{nM}$ PWTs and $1.5 \mu \mathrm{M}$ SDs.

\subsection{Preparation the $\mathbf{P}-\mathrm{L}-\mathrm{S}$ complex probes of ESDRs}

The BRCA1 gene sequence was designed on the basis of reported studies. ${ }^{33}$ Before conducting the formal experiment, $\mathrm{P}-\mathrm{L}-\mathrm{S}$ three-stranded complex probes of ESDRs were first prepared with single strands of $\mathrm{L}, \mathrm{P}$ and $\mathrm{S}$ at the molar concentration ratio of $1.2: 1: 1.2$ in a reaction buffer. Then, the solution was heated to $80{ }^{\circ} \mathrm{C}$ for $5 \mathrm{~min}$ and cooled down to room temperature gradually. Finally, the probes were stored at $4{ }^{\circ} \mathrm{C}$ for further use. 


\subsection{Fluorescence spectrophotometer analysis}

The DNA walker amplification reaction was initiated by adding $1 \mu \mathrm{L}$ of various concentrations of target gene, $12.5 \mu \mathrm{L}$ of $75 \mathrm{nM}$

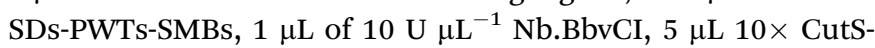
mart buffer and $30.5 \mu \mathrm{L}$ ultrapure water. The final volume was $50 \mu \mathrm{L}$. The DNA nanomachine was operated at $37^{\circ} \mathrm{C}$ for $20 \mathrm{~min}$. The supernatant was used to trigger the ESDRs at ambient temperature for $40 \mathrm{~min}$ in the solution containing $25 \mu \mathrm{L}$ of $800 \mathrm{nM}$ P-L-S and $25 \mu \mathrm{L}$ of $800 \mathrm{nM}$ assistant probe A. The ESDR products were measured by a fluorescence spectrophotometer in the wavelength range from 500 to $600 \mathrm{~nm}$ with the excitation wavelength at $492 \mathrm{~nm}$. Both the excitation and emission slits were chosen to be $5 \mathrm{~nm}$.

\subsection{Validation of the amplification strategy by gel electrophoresis}

Native polyacrylamide gel electrophoresis (PAGE) on $8 \%$ polyacrylamide gel was used to validate the designed novel fluorescent biosensor. Electrophoresis was conducted with $1 \times$ TBE buffer ( $89 \mathrm{mM}$ Tris, $89 \mathrm{mM}$ boric acid, and $2 \mathrm{mM}$ EDTA) at $\mathrm{pH}$ 8.3 and $100 \mathrm{~V}$ constant voltage for $40 \mathrm{~min}$. After this, the gel was stained with GV I for $30 \mathrm{~min}$ and then imaged using a Bio-Rad ChemDoc XRS (Bio-Rad, USA).

\section{Results and discussion}

\subsection{The principle of the cascade amplification strategy}

Our detection scheme for BRCA1 can be mainly divided into two portions: the DNA walker step and the subsequent ESDRs step. As illustrated in Scheme 1, the recognition sequence of Nb.BbvCI located on the DWTs was originally sealed by the PP. The PP involved a DNA overhang, working as a toehold. Abundant T bases were included in the DWTs as spacers to reduce steric hindrance. The DWTs partially hybridized with the PP to form PWTs. Subsequently, the PWTs were mixed with SDs at appropriate proportions and simultaneously anchored onto the SMBs through the specific streptavidin-biotin interaction. When the target BRCA1 was added to the sensing system, BRCA1 could bind with the $3^{\prime}$-terminus of the PP through the DNA toehold; this caused the release of the PP from the DWTs by TMSDR and exposed the recognition sequence of Nb.BbvCI. The intermolecular interaction between the activated DWTs and SDs enables the formation of the double stranded DNA (dsDNA) including the specific digestion site; hence, one SD is cleaved by Nb.BbvCI; this results in the dissociation of the intermediate DNA from the functionalized SMBs. Subsequently, the free DWTs spontaneously walk along the adjacent unabridged SDs to form a stable dsDNA structure and repeat the Nb.BbvCI nicking progress. In theory, one DNA walker can give rise to the release of plentiful intermediate DNA. Subsequently, the intermediate DNA, obtained by magnetic separation, specifically combines with the toehold region at the $3^{\prime}$-terminus of the strand $\mathrm{L}$ to form the $\mathrm{P}-\mathrm{L}-$ S-intermediate DNA complex and immediately catalyzes the release of the strand $\mathrm{S}$ from the three-stranded complex (P-L-S) through the ESDRs. The dissociation of the strand $\mathrm{S}$ causes the exposure of the initially obstructed toehold in the middle of the strand L. Upon the addition of the assistant probe A, the system is driven by the entropic gain of the liberated molecules to combine with the exposed toehold strand in the P-L-intermediate DNA complex to form the $\mathrm{P}-\mathrm{L}-\mathrm{A}$-intermediate DNA; this causes the liberation of the intermediate DNA and strand $\mathrm{P}$ and generation of the duplex L-A. Subsequently, the intermediate DNA is cyclically reused, whereas the strand P labeled with FAM as a signal probe is dramatically released; this leads to the enhancement of the fluorescent signal.

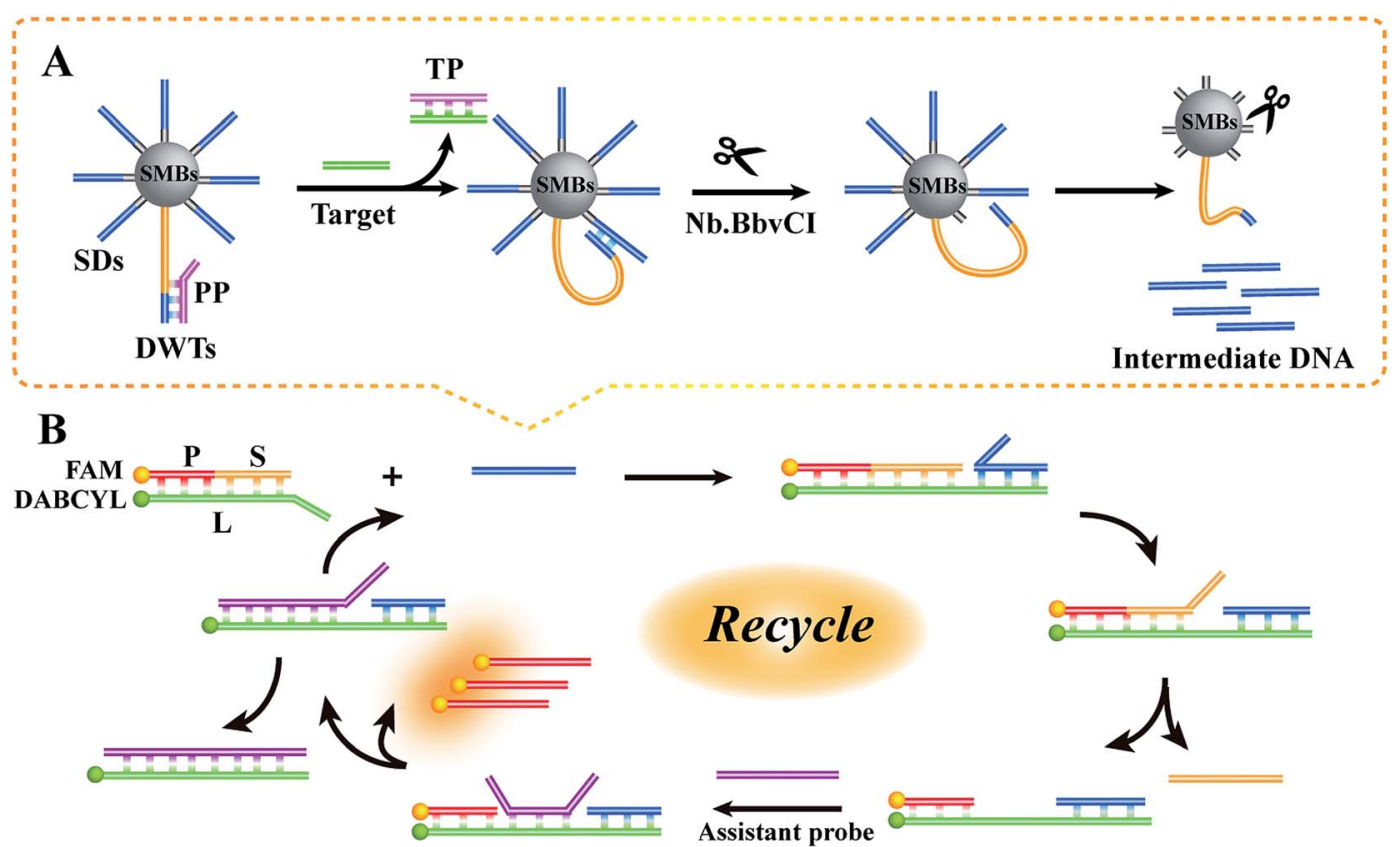

Scheme 1 Schematic of the smart fluorescent biosensor for BRCA1 gene detection. (A) 3D DNA walker machine produced intermediate DNA strands. (B) Amplification of ESDRs triggered by intermediate DNA strands and fluorescence detection. 


\subsection{Feasibility research}

At first, using the fluorescence method and native polyacrylamide gel electrophoresis, only the migration of the DNA walker was confirmed (as shown in Fig. S1†). The results showed that (1) the DWTs could be successfully activated by the target DNA and (2) the DNA walker was well established. In addition, the resulting products were detected by the fluorescence method to confirm the availability of the cascade amplification system. As shown in Fig. 1A, the fluorescence intensity of the mixture only containing $\mathrm{P}-\mathrm{L}-\mathrm{S}$ complex probes was very weak due to the quenching effect of DABCYL on FAM (curve a). The fluorescence intensity of the mixtures that lacked the target DNA, Nb.BbvCI or assistant probe A (curves b, c and d, respectively) was also very weak. In contrast, a distinct fluorescent signal was obtained from the simultaneous presence of $10 \mathrm{nM}$ of target DNA, SMBs-PWTs-SDs, $10 \mathrm{U}$ of Nb.BbvCI, $200 \mathrm{nM}$ of $\mathrm{P}-\mathrm{L}-\mathrm{S}$ complex probes and $200 \mathrm{nM}$ of assistant probe A (curve e). These results indicated that the dual-amplification strategy produced large amounts of fluorescent group-labeled signal probes on strand $P$.

To further demonstrate the validity of this strategy, native polyacrylamide gel electrophoresis was conducted. As shown in Fig. $1 \mathrm{~B}$, lane 1 represents the $\mathrm{P}-\mathrm{L}-\mathrm{S}$ complex probes. In the absence of target DNA (lane 2) or Nb.BbvCI (lane 3), only the bands of the $\mathrm{P}-\mathrm{L}-\mathrm{S}$ complex probes and assistant probe $\mathrm{A}$ were observed, indicating that no ESDRs had occurred. However, only the $\mathrm{P}-\mathrm{L}-\mathrm{S}$ complex probes could be found (lane 4) without the help of the assistant probe $\mathrm{A}$ in the whole amplification system; this might be attributed to lower concentration of the intermediate DNA. On the contrary, once all elements (10 nM of target DNA, SMBsPWTs-SDs, $10 \mathrm{U}$ of Nb.BbvCI, $1 \mu \mathrm{M}$ of $\mathrm{P}-\mathrm{L}-\mathrm{S}$ complex probes and $1 \mu \mathrm{M}$ of assistant probe A) were present simultaneously, a distinct new band (P strand) was observed (lane 5 ), which clearly confirmed that the target DNA could well initiate the DNA walker and trigger the following ESDR process.

\subsection{Optimization of the assay parameters}

To achieve the optimal detection performance of the designed fluorescent biosensor, crucial experimental variables were optimized. The value of $\Delta \mathrm{FL}\left(\Delta \mathrm{FL}=\mathrm{FL}-\mathrm{FL}_{0}\right)$ was used to estimate the biosensing performance, where $\mathrm{FL}$ and $\mathrm{FL}_{0}$ are the fluorescence intensities in the presence and absence of target DNA, respectively. As the ratio of the SDs and PWTs immobilized on the SMBs directly affected the efficiency of the DNA walker, the ratio of SDs and PWTs was first optimized. As depicted in Fig. 2A, the concentration of the SDs was set at $1500 \mathrm{nM}$; when the dosage of PWTs was shifted from 0, $25 \mathrm{nM}$, $50 \mathrm{nM}, 75 \mathrm{nM}$ to $100 \mathrm{nM}$, the fluorescence intensity increased correspondingly and reached a plateau at $75 \mathrm{nM}$. Therefore, $20: 1$ was selected as the optimal proportion for subsequent experiments.

The concentration of $\mathrm{Nb} . \mathrm{BbvCI}$ and the cleavage time were also important parameters that greatly influenced the performance of this strategy. It was observed that the $\Delta \mathrm{FL}$ intensity increased remarkably with an increase in the $\mathrm{Nb.BbvCI}$ concentration and reached a plateau at $10 \mathrm{U}$ (Fig. 2B). Moreover, the $\Delta \mathrm{FL}$ intensity increased continually with a prolonged digestion time of $\mathrm{Nb}$.BbvCI and reached a saturation value at $20 \mathrm{~min}$ (Fig. 2C). Hence, $10 \mathrm{U}$ and $20 \mathrm{~min}$ were chosen as the best conditions for the walker process for the following experiments.

In addition, the incubation time for the ESDRs was studied to improve the sensing sensitivity. The influence of the ESDR incubation time on the fluorescence signal intensity is shown in Fig. 2D. As expected, the $\Delta \mathrm{FL}$ intensity increased with an increase in reaction time and became constant at $50 \mathrm{~min}$. However, because the difference in the $\Delta \mathrm{FL}$ intensity between $40 \mathrm{~min}$ and $50 \mathrm{~min}$ was not significant, $40 \mathrm{~min}$ was chosen as the optimal condition for the ESDR process after comprehensive consideration.

\subsection{Analytical performance of the designed biosensor}

To further explore the analytical capability of our strategy, different concentrations of target DNA were monitored under the optimal conditions. As shown in Fig. 3, there was a good
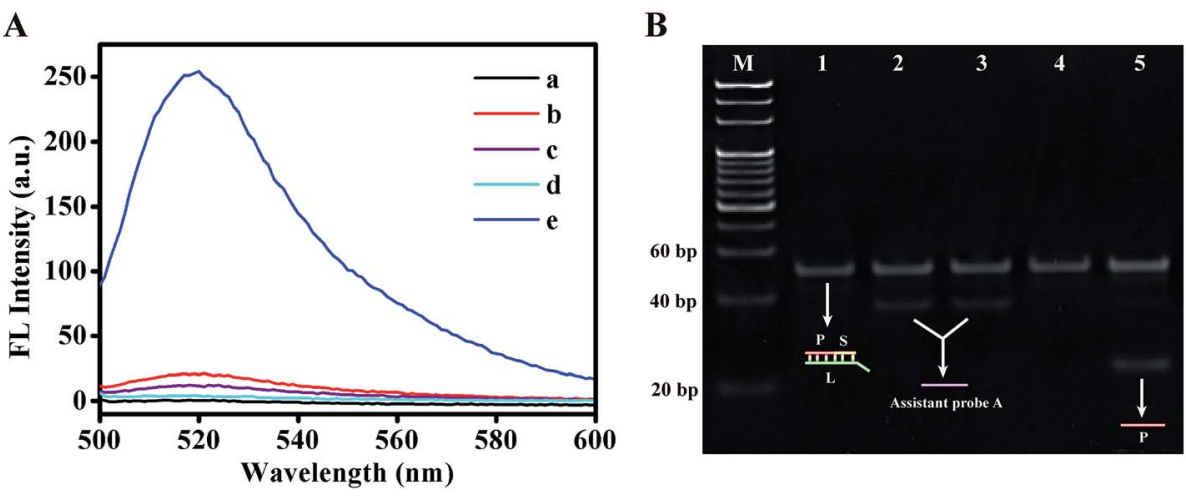

Fig. 1 (A) The biosensing strategy was analyzed by the fluorescence method. Curve a: $P-L-S$; curve b: MBs-PWTs-SDs/Nb.BbvCl/P-L-S/A; curve c: target DNA/SMBs-PWTs-SDs/P-L-S/A; curve d: target DNA/SMBs-PWTs-SDs/Nb.BbvCl/P-L-S and curve e: target DNA/SMBs-PWTsSDs/Nb.BbvCl/P-L-S/A. (B) The products were verified through 8\% Native PAGE. Lane M: 20 bp DNA ladder marker; lane 1: P-L-S; lane 2: SMBsPWTs-SDs/Nb.BbvCl/P-L-S/A; lane 3: target DNA/SMBs-PWTs-SDs/P-L-S/A; lane 4: target DNA/SMBs-PWTs-SDs/Nb.BbvCl/P-L-S; lane 5: target DNA/SMBs-PWTs-SDs/Nb.BbvCl/P-L-S/A. 10 nM target DNA was used in the experiments. 
A

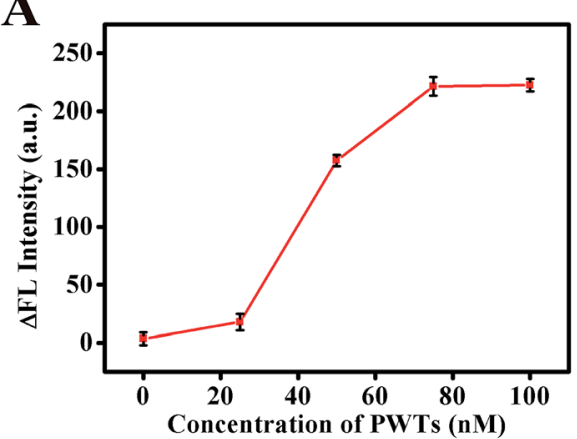

C

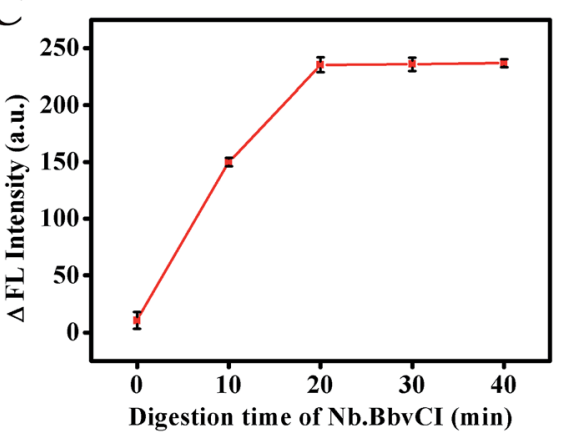

B

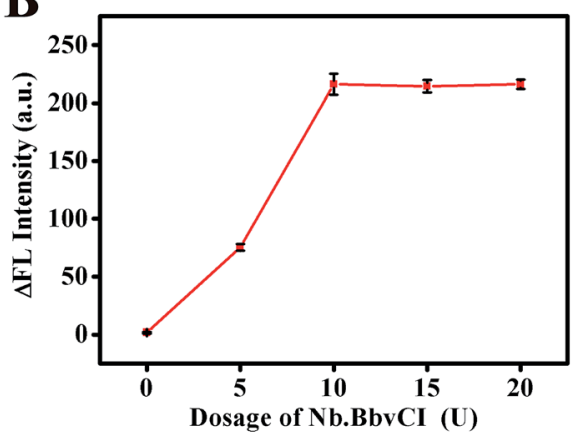

D

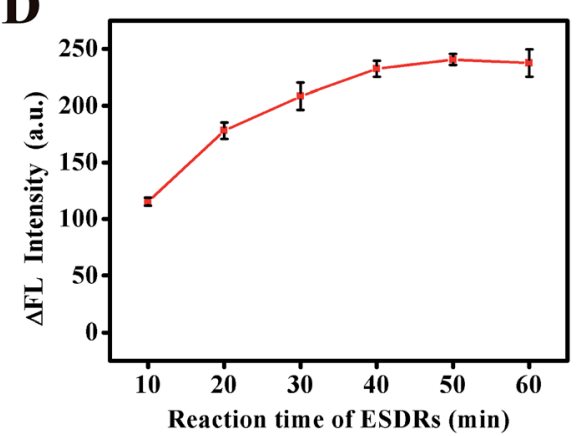

Fig. 2 Optimization of the experimental parameters: (A) the concentration of PWTs covered on the SMBs. (B) The concentration of Nb.BbvCl. (C) The digestion time of $\mathrm{Nb} . \mathrm{BbvCl}$. (D) The reaction time of ESDRs. Herein, $10 \mathrm{nM}$ target DNA was used in the experiments. Error bars represent the standard deviation of three parallel experiments.

linear relationship between the response signal and the logarithm of the target DNA concentrations in the range from 0.1 $\mathrm{pM}$ to $10 \mathrm{nM}$. The resulting linear regression equation can be expressed as $\mathrm{FL}=40.45 \log C_{\text {Target }}+87.05$ (FL indicates fluorescence signal intensity, and $C$ indicates the concentration of BRCA1) with the correlation coefficient of 0.9946. Based on the $3 \sigma$ rule, the limit of detection (LOD) for BRCA1 was calculated to be $41.44 \mathrm{fM}$. To further highlight the merits of this method, the sensitivity of this assay was compared with that of previously reported assays for the BRCA1 detection (Table S2 $\dagger$ ). The results show that the proposed robust fluorescence method has significant sensitivity and a wide dynamic concentration response range, which is attributed to the good amplification performance of the 3D DNA walker and ESDRs.

\subsection{Specificity and repeatability of this proposed strategy}

The specificity of the developed biosensor was studied by three distinct DNA sequences: P53, K-ras and PIK3CA. The concentrations of P53, K-ras and PIK3CA were $100 \mathrm{nM}$, which was 10fold that of BRCA1. As shown in Fig. 4, compared with the case of the blank control, no significant change in the signal was observed in the presence of P53, K-ras or PIK3CA. However, when BRCA1 coexisted with the other three interferences, the fluorescence intensity of the mixture was almost unchanged as compared to that when only the target DNA was present. These outcomes exhibit the remarkable specificity of this method for BRCA1 detection.

In addition, repeatability and reproducibility are important parameters for evaluating the reliability of a method and
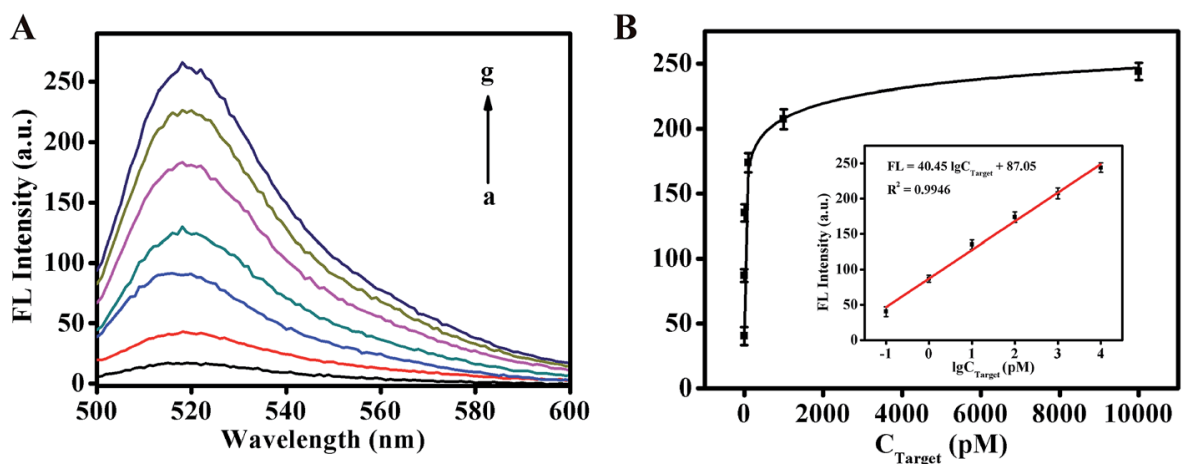

Fig. 3 (A) The fluorescent biosensor for the detection of target DNA at 0, $0.1 \mathrm{pM}, 1 \mathrm{pM}, 10 \mathrm{pM}, 0.1 \mathrm{nM}, 1 \mathrm{nM}$ and $10 \mathrm{nM}$ (from a to g). (B) The calibration curve of the designed strategy for target DNA detection. Error bars represent the standard deviation of three parallel experiments. 


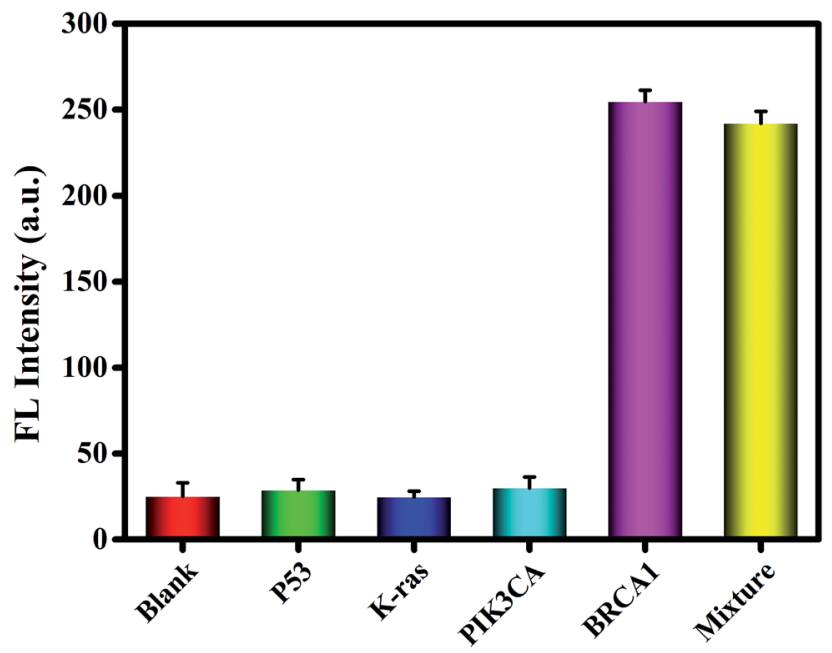

Fig. 4 Specificity test for the present biosensor for the target assay. From left to right, the samples are represented as blank, P53, K-ras, PIK3CA, BRCA1 and BRCA1 in the presence of the other interfering substances. The concentration of each interfering substance was $100 \mathrm{nM}$, whereas that of BRCA1 was $10 \mathrm{nM}$. Error bars represent the standard deviation of three parallel experiments.

therefore have also been investigated by calculating the relative standard deviation (RSD); the intra-assay and inter-assay variation have been determined by performing the assay five times with $1.0 \mathrm{nM}$ and $10 \mathrm{pM}$ target DNA; the RSD of the intra-assay results is $2.3 \%$ and $3.7 \%$ at $1.0 \mathrm{nM}$ and $10 \mathrm{pM}$, respectively. The RSD values of the inter-assays were $4.1 \%$ and $5.5 \%$ at the abovementioned concentrations of target DNA. These results indicate the reliability of this fluorescence strategy with satisfactory repeatability and reproducibility.

\subsection{Recovery test}

To further assess the capability of this sensing strategy in practical applications, the target DNA was spiked into $200 \mathrm{pg}$ $\mathrm{mL}^{-1}$ salmon sperm DNA to simulate a situation of real sample detection. In Table $\mathrm{S} 3, \uparrow$ we could see that the recoveries varied from $97.3 \%$ to $102.0 \%$, and the RSDs ranged from $5.0 \%$ to $7.5 \%$, indicating that our proposed strategy was not compromised during a complex matrix analysis. These results clearly prove that the proposed fluorescent biosensor has the potential to become a promising tool for the BRCA1 analysis.

\section{Conclusion}

In summary, we successfully constructed a cascade amplification strategy based on a 3D DNA walker machine with ESDRs. First, due to the robust manipulation capacity of the DNA walker and superior signal amplification capacity of ESDRs, as low as 0.1 pM target DNA could be detected within $1 \mathrm{~h}$. Second, the specificity of this strategy was significantly enhanced because of the rigorous sequence design of the whole system and excellent discriminative ability of TMSDR. Third, compared with common hairpin probes, our strategy fully employs linear DNA complex probes, which ensures the stability of the probes.
Fourth, the developed biosensing method has good versatility and can be extended to detect other tumor biomarkers just by changing the corresponding molecular elements. However, for the quantitative detection of real clinical samples via this biosensing method, the interference of serum matrix still needs to be overcome.

\section{Conflicts of interest}

The authors have declared that no competing interest exists.

\section{Acknowledgements}

This work was supported by the National Natural Science Foundation of China (81371904 and 81873980).

\section{References}

1 R. C. Qian, Y. Cao, L. J. Zhao, Z. Gu and Y. T. Long, Angew. Chem., Int. Ed., 2017, 56, 4802-4805.

2 J. K. Awino, S. Gudipati, A. K. Hartmann, J. J. Santiana, D. F. Cairns-Gibson, N. Gomez and J. L. Rouge, J. Am. Chem. Soc., 2017, 139, 6278-6281.

3 J. S. Wang, Y. H. Yan and D. Y. Zhang, Nat. Chem., 2017, 9, 1222-1228.

4 K. Lee, Y. Cui, L. P. Lee and J. Irudayaraj, Nat. Nanotechnol., 2014, 9, 474-480.

5 M. Liu, W. Q. Zhang, Q. Zhang, J. D. Brennan and Y. F. Li, Angew. Chem., Int. Ed., 2015, 54, 9637-9641.

6 Q. Q. Liu, J. Q. Liu, D. G. He, T. P. Qing, X. X. He, K. M. Wang and Y. F. Mao, RSC Adv., 2016, 6, 90310-90317.

7 F. A. Wang, J. Elbaz, R. Orbach, N. Magen and I. Willner, J. Am. Chem. Soc., 2011, 133, 17149-17151.

8 C. Li, Y. C. Yang, B. Zhang, G. F. Chen, Z. X. Wang and G. X. Li, Part. Part. Syst. Charact., 2014, 31, 201-208.

9 F. A. Wang, C. H. Lu, X. Q. Liu, L. N. Freage and I. Willner, Anal. Chem., 2014, 86, 1614-1621.

10 Y. Chen, M. L. Yang, Y. Xiang, R. Yuan and Y. Q. Chai, Anal. Chim. Acta, 2013, 796, 1-6.

11 Y. S. Jiang, B. L. Li, J. N. Milligan, S. Bhadra and A. D. Ellington, J. Am. Chem. Soc., 2013, 135, 7430-7433.

12 M. W. Wang, Y. Y. Tang, Y. Y. Chen, Y. Cao and G. F. Chen, Anal. Chim. Acta, 2019, 1045, 77-84.

13 M. H. Liu, J. L. Fu, C. Hejesen, Y. H. Yang, N. W. Woodbury, K. Gothelf, Y. Liu and H. Yan, Nat. Commun., 2013, 4, 21272131.

14 M. Qing, S. B. Xie, W. Cai, D. Y. Tang, Y. Tang, J. Zhang and R. Yuan, Anal. Chem., 2018, 90, 11439-11445.

15 C. Jung, P. B. Allen and A. D. Ellington, Nat. Nanotechnol., 2016, 11, 157-163.

16 Y. H. Ji, L. Zhang, L. Y. Zhu, J. P. Lei, J. Wu and H. X. Ju, Biosens. Bioelectron., 2017, 96, 201-205.

17 L. D. Wang, R. J. Deng and J. H. Li, Chem. Sci., 2015, 6, 67776782.

18 X. S. Yang, D. M. Shi, S. M. Zhu, B. J. Wang, X. J. Zhang and G. F. Wang, ACS Sens., 2018, 3, 1368-1375. 
19 X. M. Qu, D. Zhu, G. B. Yao, S. Su, J. Chao, H. J. Liu, X. L. Zuo, L. H. Wang, J. Y. Shi, L. H. Wang, W. Huang, H. Pei and C. H. Fan, Anal. Chim. Acta, 2017, 56, 1855-1858.

20 Z. Q. Xu, L. L. Liao, Y. Q. Chai, H. J. Wang and R. Yuan, Anal. Chem., 2017, 89, 8282-8287.

21 X. L. Yang, Y. N. Tang, S. D. Mason, J. B. Chen and F. Li, ACS Nano, 2016, 10, 2324-2330.

22 J. G. Xu, Z. S. Wu, H. L. Li, Z. M. Wang, J. Q. Le, T. T. Zheng and L. E. Jia, Biosens. Bioelectron., 2016, 86, 1024-1030.

23 X. Y. Wu, Y. Q. Chai, P. Zhang and R. Yuan, ACS Appl. Mater. Interfaces, 2015, 7, 713-720.

24 Y. Zhang, L. X. Wang, F. Luo, B. Qiu, L. H. Guo, Z. Q. Weng, Z. Y. Lin and G. N. Chen, Chem. Commun., 2017, 53, 28852992.

25 R. Wang, L. Wang, X. W. Xu and W. Jiang, Sens. Actuators, B, 2018, 268, 287-292.
26 H. P. Wu, X. Y. Zhou, W. Cheng, T. X. Yuan, M. Zhao, X. L. Duan and S. J. Ding, Analyst, 2018, 143, 4974-4980.

27 D. M. Huang, X. M. Li, B. Shen, J. Li, X. J. Ding, X. Y. Zhou, B. Guo, W. Cheng and S. J. Ding, Anal. Chim. Acta, 2018, 273, 393-399.

28 D. Y. Zhang, A. J. Turberfield, B. Yurke and E. Winfree, Science, 2007, 318, 1121-1125.

29 Y. Q. Wu, D. Y. Zhang, P. Yin and F. Vollmer, Small, 2014, 10, 2067-2076.

30 K. Zhang, K. Wang, X. Zhu and M. H. Xie, Anal. Chim. Acta, 2017, 949, 53-58.

31 Q. M. Feng, Y. H. Guo, J. J. Xu and H. Y. Chen, ACS Appl. Mater. Interfaces, 2017, 9, 17637-17644.

32 S. Ou, T. Xu, X. Liu, X. Y. Yu, R. Li, J. Deng, J. X. Yuan and Y. M. Chen, Sens. Actuators, B, 2018, 255, 3057-3063.

33 X. J. Qin, S. X. Xu, L. Deng, R. F. Huang and X. F. Zhang, Biosens. Bioelectron., 2016, 85, 957-963. 